\title{
In-vitro fermentation of whole seaweed and a polysaccharide-rich extract derived from the edible red seaweed Palmaria palmate
}

\author{
P. Cherry ${ }^{1}$, S. Yadav ${ }^{2}$, C. O’Callaghan ${ }^{3}$, Z.A. Popper ${ }^{3}$, R.P. Ross ${ }^{4}$, E.M. McSorley ${ }^{1}$, \\ P.J. Allsopp ${ }^{1}$ and C. Stanton ${ }^{2,4}$ \\ ${ }^{1}$ Northern Ireland Centre for Food and Health, Ulster University, Coleraine, BT52 1SA, Northern Ireland, ${ }^{2}$ Teagasc \\ Food Research Centre, Moorepark, Co. Cork, Republic of Ireland, ${ }^{3}$ Ryan Institute, National University of Ireland \\ Galway, Ireland and ${ }^{4}$ Alimentary Pharmabiotic Centre, University College Cork, Republic of Ireland
}

Dietary fibre is considered an important component of a healthy diet, with evidence indicating that fibre may positively modulate gut microbiota composition and metabolism ${ }^{(1)}$. There is increased attention upon the potential health benefits of seaweeds due to high fibre content ${ }^{(2)}$, where the commonly consumed red seaweed Palmaria palmata is of particular interest due to the presence of the fermentable fibre Xylan, previously shown to exert prebiotic effects ${ }^{(3)}$. This study aims to provide initial indications of the impact of Palmaria palmata and its polysaccharide fibre component on the composition of the human microbiota.

This study used faecal batch culture models to assess the in-vitro fermentability of pre-washed, freeze dried, whole seaweed (WS) and polysaccharide-rich (PR) extracts of Palmaria palmata, alongside Synergy 1 (positive control) and cellulose (negative control). Treatments underwent an in-vitro simulated upper gastrointestinal digestion process followed by a 48 hour in-vitro batch culture faecal fermentation (1\% w/v treatment). Enumeration of total bacteria, Lactobacilli and Bifidobacteria was determined using qPCR as well as culture based methods at all time points $(\mathrm{t}=0,5,10,24,36$ and 48 hours). Data was log-transformed prior to two-way ANOVA statistical analysis $(\mathrm{n}=3)$.

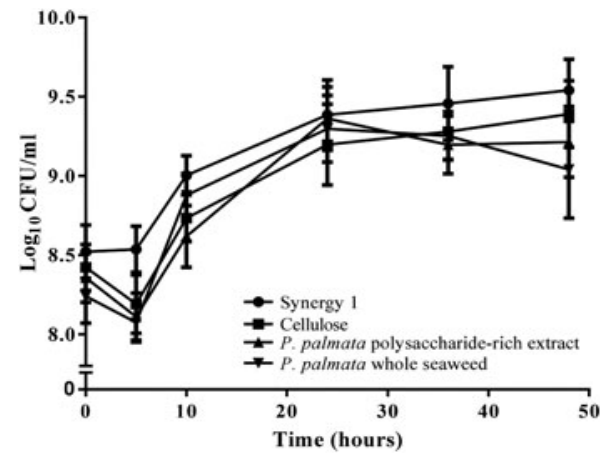

Fig. 1. Total bacteria.

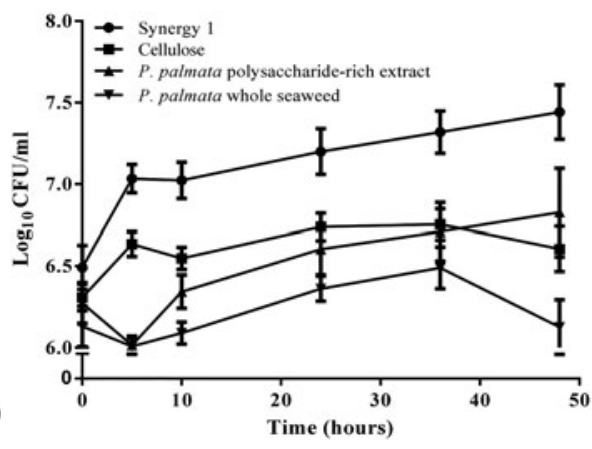

Fig. 2. Lactobacilli.

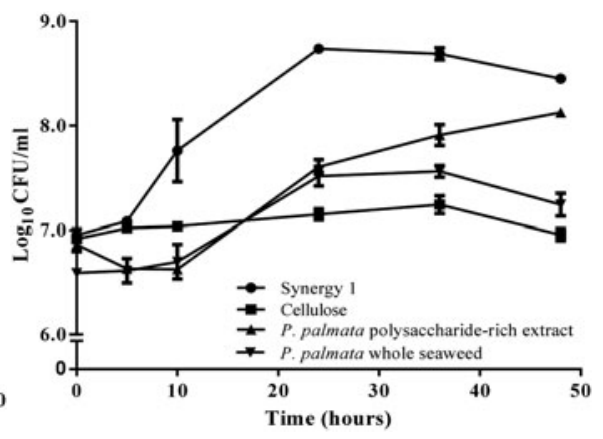

Fig. 3. Bifidobacteria.

qPCR analysis (Fig. 1-3): No significant differences were observed in total bacterial counts between substrates at any time point (Fig. 1; $P>0 \cdot 1)$. Neither WS nor PR treatments showed a significant increase in Lactobacilli relative to cellulose (Fig. $2 ; P>0 \cdot 1)$. WS triggered a significant increase in Bifidobacteria relative to cellulose at $24 \mathrm{~h}$ and $36 \mathrm{~h}$ (Fig. $3 ; P<0 \cdot 05)$, whilst PR was shown to have a stimulatory effect at $24 \mathrm{~h}, 36 \mathrm{~h}$ and $48 \mathrm{~h}(P<0 \cdot 001)$. Further, PR samples were analogous to Synergy 1 at $48 \mathrm{~h}(P>0 \cdot 1)$. Similar trends were observed in the culture based analysis.

These data suggest that freeze dried Palmaria palmata powder and polysaccharide-rich extracts of Palmaria palmata exhibit bifidogenic activity. Metagenomic sequencing of the microbial population and targeted metabolomics is required to verify these findings.

1. Simpson HL \& Campbell BJ (2015) Aliment Pharmacol Ther 42, 158-179.

2. MacArtain P, Gill CIR, Brooks M et al. (2007) Nutrition Reviews 65, 535-543.

3. O'Sullivan L, Murphy B, McLoughlin P et al. (2010) Marine Drugs 8, 2038-2064. 